\title{
Distal transradial access in the anatomical snuffbox for diagnostic cerebral angiography
}

\author{
Marie-Christine Brunet, ${ }^{1}$ Stephanie H Chen, ${ }_{1}^{1}$ Samir Sur, ${ }^{2}$ David J McCarthy, ${ }^{3}$ \\ Brian Snelling, ${ }^{2}$ Dileep R Yavagal, ${ }_{1}^{4}$ Robert M Starke, ${ }^{1,5}$ Eric C Peterson ${ }^{2}$
}

${ }^{1}$ Department of Neurological Surgery, University of Miami School of Medicine, Miami, Florida, USA

${ }^{2}$ Neurological Surgery, University of Miami MILLER School of Medicine, Miami, Florida, USA ${ }^{3}$ Neurosurgery, University of Miami School of Medicine, Miami, Florida, USA ${ }^{4}$ Neurology and Neurosurgery, University of Miami, Miami, Florida, USA

${ }^{5}$ University of Miami School of Medicine, Miami, Florida, USA

\section{Correspondence to} Dr Eric C Peterson, Neurological Surgery, University of Miami Miller School of Medicine, Miami FL 33136, USA; ericpete@med.miami.edu

Received 10 January 2019 Revised 29 January 2019 Accepted 30 January 2019 Published Online First 27 February 2019

\section{ABSTRACT}

Background The transradial approach for endovascular angiography and interventional procedures is superior to the traditional transfemoral approach in several metrics, including lower access-site complication rates, higher patient satisfaction, and lower hospital costs. Interventional cardiologists have begun to adopt the distal transradial approach (dTRA) for coronary interventions as it has an improved safety profile and improved procedural ergonomics. Adaptation of dTRA for neuroendovascular procedures promises similar benefit, but requires a learning curve.

Objective To report the first use of dTRA for diagnostic cerebral angiography and demonstrate the feasibility and safety of a dTRA.

Methods A retrospective review of our prospective institutional database of consecutive cases of cerebral DSA performed via dTRA between August 2018 and December 2018 was performed. Patient demographics, procedural and radiographic metrics, and clinical data were recorded.

Results 85 patients were identified with an average age of 53.8 years (range 18-82); $67(78.8 \%$ ) patients were female. 78 patients underwent successful dTRA diagnostic cerebral angiography, with a mean of five vessels catheterized and average fluoroscopy time of $12.0 \mathrm{~min}$, or $2.6 \mathrm{~min}$ for each vessel. Seven patients required conversion to transfemoral access, with the most common reason being inability to advance the wire and radial artery spasm. There were no complications.

Conclusion ITRA is associated with decreased rates of radial artery occlusion, ischemic hand events, as well as improved patient comfort, faster periprocedural management, and cost benefits. Our preliminary experience with ITRA for diagnostic cerebral angiography demonstrates excellent feasibility and safety in combination with relative efficiency.

\section{INTRODUCTION}

Radial access for coronary and peripheral interventional angiography has dramatically increased worldwide since its introduction in 1989 by Campeau. ${ }^{1}$ Interventional cardiology studies comparing a transradial approach (TRA) with a transfemoral approach (TFA) have shown an unequivocal benefit of the former, with reduced morbidity and mortality, greater patient preference, and cost reductions. ${ }^{2-13}$ The Matrix Trial of over 8400 patients demonstrated a significant relative risk reduction of 0.67 for major bleeding and a significant relative risk reduction of 0.72 for all-cause mortality. ${ }^{14}$

Interventional cardiologists have recently begun to adopt a modification of TRA, the distal transradial approach (dTRA), for coronary interventions. ${ }^{15}$ A rare minor complication of TRA is radial artery occlusion (RAO), a clinically silent complication that occurs in approximately $4 \%$ of patients. ${ }^{16}$ Repeated transradial procedures may increase the occurrence of arterial occlusion and limit the viability of the vessel as an access site for further procedures or grafting. The dTRA confers theoretical improvements in preservation of the radial artery, as well as ease of left-sided catheterization, and improved patient-operator ergonomics. More importantly, this segment of the radial artery is distal to the origin of the superficial palmar branch, which supplies numerous palmar collaterals to the deep palmar arch. Puncture at this site preserves palmar collaterals, decreasing the risk of ischemic hand events even in cases of postprocedural arterial occlusion at the access site. Preliminary cardiology results have shown technical and procedural success and no access-site complications. ${ }^{17-20}$ We recently reported the first use of distal radial artery access for neurointervention in two cases, but no studies in the English literature have examined the efficacy, safety, or learning curve of dTRA for neurointerventions, which include additional challenges of accessing and navigating tortuous and small-caliber arteries. $^{21}$ In this study, we present our initial experience with carrying out diagnostic digital subtraction angiography (DSA) via the dTRA approach.

\section{METHODS}

After receiving institutional review board approval, we retrospectively analyzed our prospective institutional database of cerebral DSA cases performed via dTRA between August 2018 and December 2018. Patient demographics, procedural and radiographic metrics, and clinical data were recorded.

\section{Distal transradial approach technique}

Procedural consent, preprocedure testing, room setup, and postprocedure assessment are performed as previously described with standard TRA. $^{22-26}$ Patients with a history of right arm trauma, surgery, known anatomical variants, arterial occlusion, or stenosis were excluded. In contrast to the traditional transradial technique the puncture site is distal to the palmer arch, and thus, preprocedural assessment of collateral palmar circulation 
via Barbeau testing is not applicable for dTRA. ${ }^{27} 28$ The decision for dTRA was at the discretion of the treating physician. All procedures, including all aspects of the angiography, access and closure, were performed by fellows or attending physicians who were well-versed in both standard TRA and TFA techniques, having obtained $>50$ angiograms via each approach. Two operators performed the vast majority of cases.

In the preoperative holding area, topical 2.5\% lidocaine/ prilocaine cream is applied to the anatomical snuffbox region of the wrist and covered with an adhesive dressing for 15-30 min before the start of the procedure. The patient is positioned with the hand tightly against the hip. Bolsters are stacked inferior and caudal to the hand to allow a consistent platform on which the catheters and wires can rest. Notably, in contrast to traditional TRA, for dTRA the hand is allowed to lie in its natural position with the palm facing the hip. A slight ulnar abduction is achieved by curling the thumb into the other four fingers, thus forcing the fossa radialis upwards. A radial drape is used with a standard femoral angiography drape overlaid. Once in a place, a window is cut into the femoral angiography drape to expose the wrist.

Ultrasound guidance for radial access was shown to improve the success and speed of cannulation in the RAUST Trial and was thus employed for all cases. ${ }^{29}$ The periarterial tissue is infiltrated with $2 \mathrm{~mL}$ of lidocaine. A 20 gauge needle is angled $45-50$ degrees medially in the direction of the radial artery and the anterior wall of the artery is punctured. On brisk blood return, a 0.025 hydrophilic guidewire is threaded into the radial artery, and a $10 \mathrm{~cm} 5 \mathrm{~F}$ Glidesheath Slender (Terumo, Japan) is placed into the radial artery. Antispasmolytic agents (verapamil $2.5 \mathrm{mg}$ and nitroglycerin $200 \mu \mathrm{g}$ ) are infused in the sheath and $4000 \mathrm{U}$ of heparin are administered intravenously. Similar to TFA, access is obtained with only the anteroposterior plane in position. As the arm is closer to the body than with standard TRA, rotation of the table is usually not necessary to visualize the right forearm under anteroposterior monoplane. The remainder of the case proceeds in an identical fashion to standard TRA. ${ }^{22}$

Closure is performed with the Safeguard closure device (Merit, Salt Lake City, Utah, USA). For reasons expanded on in the 'Discussion' section, true patent hemostasis documented with plesthysmography is not possible. However, after inflation, we slowly release small amounts of air from the device until a small amount of bleeding occurs, after which we inject another 1-2 cc of air. By using the minimum amount of compression needed for hemostasis, we maximize the chances of preserved radial artery patency.

\section{RESULTS}

From the period of August 2018 to December 2018, 85 dTRA procedures were performed. The right distal radial artery was accessed in $100 \%$ of cases. In all cases, the arteries of interest were able to be catheterized. The median number of vessels catheterized for angiography was five (range 1-6). Seven patients $(8.2 \%)$ required conversion to standard TRA (one patient) or TFA (six patients) owing to artery spasm or an inability to cannulate the artery. We did not encounter radial artery spasm after initial access in any cases and no additional spasmolytic medication was necessary. The mean radiation time was $12.0 \mathrm{~min}$ with a mean radiation time per vessel of $2.6 \mathrm{~min}$. Notably, in the first and second quarters of our experience, $14.3 \%$ of cases were converted to traditional TRA or TFA. However, in the third quarter our failure rate decreased to $4.7 \%$ and $0 \%$ in the last (fourth) quarter (figure 1). The mean distal radial artery diameter was $2.4 \mathrm{~mm}$ (range $1.4-4 \mathrm{~mm}$ ) and the mean distal forearm radial artery diameter was $2.7 \mathrm{~mm}$ (range $1.4-4.2 \mathrm{~mm}$ ) (table 1).

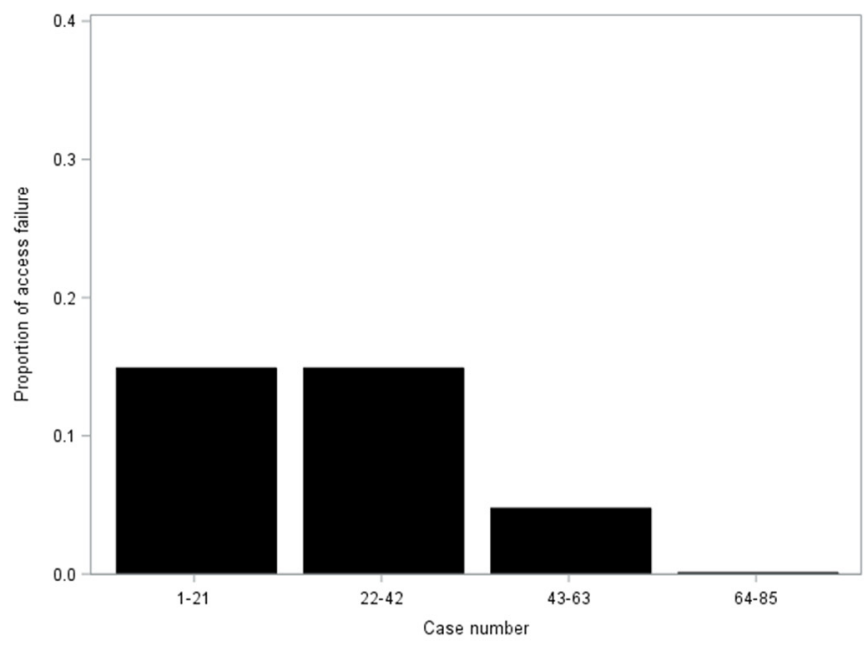

Figure 1 Failure rate divided into four quarters.

Conversion to TFA was required in three additional patients because of arteria lusoria configuration, an anatomical variant found in $0.2-1.7 \%$ of patients, which precludes catheterization of the great vessels via a right transradial approach. ${ }^{30}$ Those three cases are not included in the access failure rate as the distal radial artery was cannulated and navigated without difficulty.

\section{Statistical analysis}

Data are presented as mean and SD for normal continuous variables, median and range for non-parametric continuous variables, and as frequency for categorical variables. Statistics of means were carried out using an unpaired Student t-test, with and without equal variance (Levene's test) as necessary, and Wilcoxon rank sum tests when variables were not normally distributed. Statistical analyses of categorical variables were carried out using $\chi^{2}$ and Fisher's exact tests, as appropriate. Statistical analysis was carried out with S.A.S 9.4 (Cary, North Carolina, USA).

\section{DISCUSSION}

Owing to the clear safety benefits of transradial access, our center uses a default traditional transradial approach for diagnostic angiography. However, recent reports in the interventional cardiology literature have suggested additional advantages of decreased rates of RAO and ischemic hand events, as well as improved ergonomics with distal transradial artery access in the 'snuffbox'. ${ }^{17-20}$ The anatomical snuffbox is a depressed space located in the radial part of the wrist and surrounded laterally by the tendons of the abductor pollicis longus and extensor pollicis

\begin{tabular}{lc}
\hline Table 1 & \\
\hline Characteristics & $\mathrm{n}=85$ \\
\hline Age (years), mean (SD) & $53.8(15)$ \\
\hline Male, $\mathrm{n}(\%)$ & $18(21.2)$ \\
\hline Vessels catheterized, median (IQR) & $5(1-6)$ \\
\hline Fluoroscopic time (min), mean (SD) & $12.0(5.7)$ \\
\hline Fluoroscopic time per vessel (min), mean (SD) & $2.6(1.4)$ \\
\hline Distal radial size (mm), mean (SD)* & $2.4(0.6)$ \\
\hline Radial size (mm), mean (SD)* & $2.7(0.6)$ \\
\hline Access failure, $\mathrm{n}(\%)$ & $7(8.2)$ \\
\hline *Values available for 46 patients.
\end{tabular}




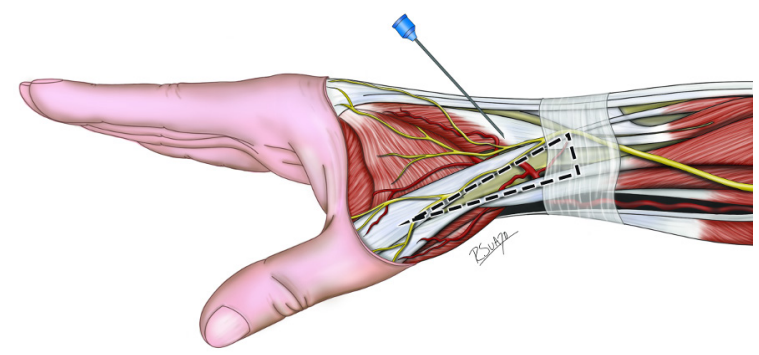

Figure 2 Positioning of hand and anatomical landmarks for distal radial artery puncture (snuffbox area).

brevis muscles and medially by the tendon of the extensor pollicis longus muscle. The distal radius, scaphoid, trapezium, and the base of the first metacarpal bone constitute the base of this triangular area (figure 2). The radial artery bifurcates before entering the snuffbox, giving off the superficial palmar branch, which forms anastomoses with the ulnar artery. The distal part of the radial artery continues through the snuffbox and travels dorsally to supply the deep palmar arch of the hand. The notable feature of the distal radial artery segment is its location distal to the origin of the superficial palmar branch of the radial artery, which forms significant collaterals with the deep palmar arch (figure 3).

Although the rate of RAO in standard TRA is low (1-6\%) and nearly always asymptomatic, rare cases of hand ischemia have been described in the setting of inadequate ulnar collateral circulation, as well as symptoms of pain or paresthesias at the site of the arterial occlusion. ${ }^{16}$ Additionally,

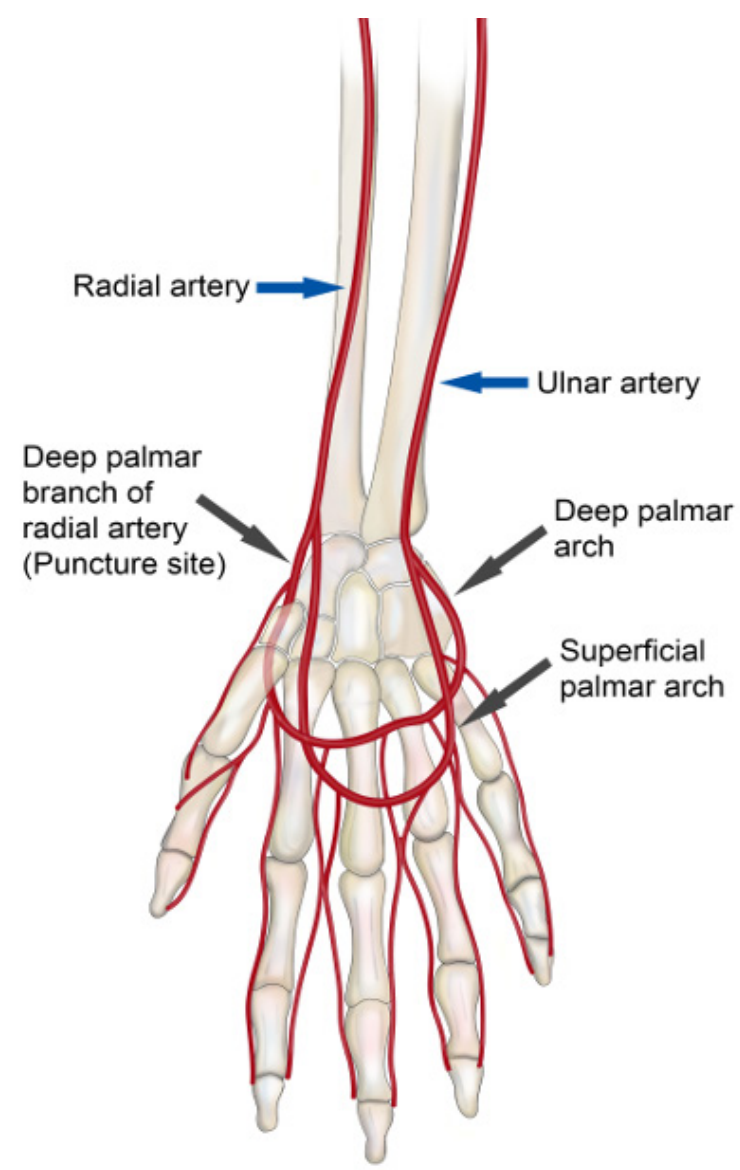

Figure 3 Vascular anatomy of the hand. chronic RAO or intimal damage to the radial artery can limit future transradial access as well as use of the radial artery as a conduit for bypass grafting or arteriovenous fistula formation. Puncture distal to the origin of the superficial palmar branch of the radial artery diminishes the risk of ischemic events in the setting of RAO, ${ }^{31}$ because postprocedural RAO typically occurs at the site of puncture. Owing to bifurcation of the radial artery, in dTRA the superficial palmar branch is preserved and allows for adequate perfusion of the hand even in the event of distal radial RAO. Furthermore, RAO in the distal radial artery does not limit future interventions via a traditional TRA or surgical grafting. One randomized study of 200 patients demonstrated no significant difference in rates of RAO after dTRA versus TRA for coronary intervention (5\% dTRA vs 9\% TRA, $\mathrm{p}=0.407) .{ }^{32}$ However, in a study of 1320 patients who underwent right dTRA for coronary intervention, late RAO was observed in only $0.61 \%$ of cases. ${ }^{18}$ There were no symptomatic events associated with RAO in either study.

Despite the bifurcation of the radial artery before the anatomical snuffbox, we found no significant difference in the size of the distal radial artery (snuffbox) versus the conventional forearm radial artery ( $2.5 \mathrm{~mm}$ vs $2.9 \mathrm{~mm}$ ). Consequently, we were able to use the same 5 Fr sheath and catheters routinely used for TRA diagnostic cerebral angiography. In addition, as opposed to the full supination and extension of the wrist necessary for traditional TRA, the neutral resting position of the arm with dTRA is more comfortable for patients, especially when awake for diagnostic angiography.

Interest in dTRA in interventional cardiology has largely been driven by improved ergonomics. Left-sided approaches are commonly used for cardiac catheterization, but can be cumbersome for the operator who stands on the right side of the patient. The natural hand position of dTRA allows for simple left-sided dTRA with the hand draped across the body, thus making access easier for the interventionalist standing on the patient's right side. Preferable leftsided trajectory to the coronary vessels has led to the rapid adoption of this technique in interventional cardiology. By contrast, the utility of left-sided dTRA is limited in neurointerventions where a right-sided trajectory is typically more advantageous for catheterizing the cerebral vessels, and even the left vertebral artery can easily be accessed from the right radial artery. Nevertheless, this option may be particularly useful in cases where the left vertebral artery is dominant or in cases of a challenging vertebral artery origin angle from TFA or right TRA. Additionally, left-sided dTRA may be considered in patients who require urgent left vertebral artery endovascular access in the setting of stroke or vertebral artery dissection.

Lastly, Koutouzis et al found a significantly shorter postprocedural duration of hemostasis $(568 \pm 462 \mathrm{~s}$ vs $841 \pm 574 \mathrm{~s}$, $\mathrm{p}=0.002$ ) as well as slightly higher rates of patient satisfaction in the dTRA group than in the TRA group, although this difference was not significant. ${ }^{32}$

Data on distal radial access remain limited in both the cardiology and neurointerventional literature. Failure rates requiring cross over to either TRA or TFA are reported to be between $0.3 \%$ and $11 \% .^{15}{ }^{17-20} 32$ Our failure rate was $8 \%$, with the majority of our failed dTRA cases converted to TFA for convenience, as the arm would have to be repositioned with more supination and subsequently reprepped and draped to perform rescue TRA. Additionally, on a rare occasion, repetitive manipulation of the wire in the distal radial artery led to prohibitive 
vasospasm more proximally after failure. Similar to our initial experience with TRA for diagnostic angiography, we have had a substantial learning curve with dTRA. Owing to the more angulated trajectory of the distal radial artery as compared with the straight trajectory of the proximal radial artery, initially we were unable to insert the wire, despite brisk blood flow from the cannula indicating we were in the lumen of the vessel. However, we found that aiming the needle 45-50 degrees medially toward the course of the distal radial artery (which can be traced with ultrasound) corrected that problem. Koutouzis et al reported similar observations and reported a significantly longer time of cannulation in the dTRA group than in the TRA group $(269 \pm 251 \mathrm{~s}$ vs $140 \pm 161 \mathrm{~s}, \mathrm{p}<0.001)$ although this did not affect overall procedure time. ${ }^{32}$ However, it is important to note the number of failed attempts at dTRA decreased steeply with experience (figure 1).

Owing to the lack of dedicated radial catheters at this time, an additional disadvantage of dTRA, which may rarely be encountered, is that the length of the catheter may be too short. Given that the snuffbox artery is approximately $3-4 \mathrm{~cm}$ below the common radial entry site, operators may find the rare problem of insufficient catheter length, particularly in very tall and tortuous patients.

\section{CONCLUSIONS}

Distal TRA is a modification of traditional TRA that offers advantages of improved patient comnfort, lower risk of RAO, and improved ergonomics for a left radial approach. We found the approach had several advantages for diagnostic cerebral angiography, although with a learning curve for the access. Given our preliminary success, we have transitioned our diagnostic cerebral angiography practice from traditional TRA primarily to dTRA. We found similar radial artery sizes between TRA and dTRA in our series, but further clinical investigation is warranted to determine whether large-bore interventions can routinely be performed via this approach.

Contributors M-CB, SHC, RMS, ECP: substantial contributions to the conception or design of the work and the acquisition, analysis, and interpretation of data for the work; drafting the work or revising it critically for important intellectual content; final approval of the version to be published; and agreement to be accountable for all aspects of the work in ensuring that questions related to the accuracy or integrity of any part of the work are appropriately investigated and resolved. SS: substantial contributions to the conception or design of the work and drafting the work or revising it critically for important intellectual content; final approval of the version to be published; agreement to be accountable for all aspects of the work in ensuring that questions related to the accuracy or integrity of any part of the work are appropriately investigated and resolved. DJMCC: substantial contributions to the conception or design of the work and analysis, interpretation of data for the work; drafting the work or revising it critically for important intellectual content; final approval of the version to be published; agreement to be accountable for all aspects of the work in ensuring that questions related to the accuracy or integrity of any part of the work are appropriately investigated and resolved. BS, DRY: substantial contributions to the conception or design of the work and interpretation of data for the work; drafting the work or revising it critically for important intellectual content; final approval of the version to be published; agreement to be accountable for all aspects of the work in ensuring that questions related to the accuracy or integrity of any part of the work are appropriately investigated and resolved. We would like to thank Mr. Roberto Suazo for his graphic design expertise in producing our manuscript figures.

Funding The authors have not declared a specific grant for this research from any funding agency in the public, commercial or not-for-profit sectors.

Competing interests DRY: Medtronic Neurovascular: consultant; Cerenovus: consultant; Rapid Medical and Neuralanalytics: consultant. RMS: Medtronic Neurovascular: consultant; Penumbra: consultant; Cerenovus: consultant; Abbott: consultant. ECP: Stryker Neurovascular: consultant; Penumbra: consultant; RIST Neurovascular: stockholder; Medtronic Neurovascular: consultant; Cerenovus: consultant. BS: RIST Neurovascular: stockholder.
Patient consent for publication Not required.

Provenance and peer review Not commissioned; externally peer reviewed.

\section{REFERENCES}

1 Campeau L. Percutaneous radial artery approach for coronary angiography. Cathet Cardiovasc Diagn 1989;16:3-7.

2 Mitchell MD, Hong JA, Lee BY, et al. Systematic review and cost-benefit analysis of radial artery access for coronary angiography and intervention. Circ Cardiovasc Qual Outcomes 2012;5:454-62.

3 Mann JT, Cubeddu MG, Schneider JE, et al. Right radial access for PTCA: a prospective study demonstrates reduced complications and hospital charges. J Invasive Cardiol 1996;8 Suppl D:40-4

4 Brueck M, Bandorski D, Kramer W, et al. A randomized comparison of transradial versus transfemoral approach for coronary angiography and angioplasty. JACC Cardiovasc Interv 2009:2:1047-54

5 Caputo RP, Tremmel JA, Rao S, et al. Transradial arterial access for coronary and peripheral procedures: executive summary by the Transradial Committee of the SCAI. Catheter Cardiovasc Interv 2011;78:823-39.

6 Chase AJ, Fretz EB, Warburton WP, et al. Association of the arterial access site at angioplasty with transfusion and mortality: The m.O.R.T.A.L study (mortality benefit of reduced transfusion after percutaneous coronary intervention via the arm or leg). Heart 2008;94:1019-25.

7 Jolly SS, Yusuf S, Cairns J, et al. Radial versus femoral access for coronary angiography and intervention in patients with acute coronary syndromes (RIVAL): a randomised, parallel group, multicentre trial. Lancet 2011;377:1409-20.

8 Jolly SS, Amlani S, Hamon M, et al. Radial versus femoral access for coronary angiography or intervention and the impact on major bleeding and ischemic events: a systematic review and meta-analysis of randomized trials. Am Heart $J$ 2009;157:132-40.

9 Wang YB, Fu XH, Wang XC, et al. Randomized comparison of radial versus femoral approach for patients with STEMI undergoing early $\mathrm{PCI}$ following intravenous thrombolysis. J Invasive Cardiol 2012;24:412-6.

10 Alnasser SM, Bagai A, Jolly SS, et al. Transradial approach for coronary angiography and intervention in the elderly: a meta-analysis of 777,841 patients. Int J Cardiol 2017:228:45-51.

11 Kok MM, Weernink MGM, von Birgelen C, et al. Patient preference for radial versus femoral vascular access for elective coronary procedures: the PREVAS study. Catheter Cardiovasc Interv 2018:91:17-24.

12 Kolkailah AA, Alreshq RS, Muhammed AM, et al. Transradial versus transfemoral approach for diagnostic coronary angiography and percutaneous coronary intervention in people with coronary artery disease. Cochrane Database Syst Rev 2018;4:CD012318

13 Mamas MA, Tosh J, Hulme W, et al. Health economic analysis of access site practice in England during changes in practice: Insights from the British Cardiovascular Interventional Society. Circ Cardiovasc Qual Outcomes 2018;11:e004482.

14 Valgimigli M, Gagnor A, Calabró P, et al. Radial versus femoral access in patients with acute coronary syndromes undergoing invasive management: a randomised multicentre trial. Lancet 2015;385:2465-76.

15 Kiemeneij F. Left distal transradial access in the anatomical snuffbox for coronary angiography (IdTRA) and interventions (IdTRI). Eurolntervention 2017;13:851-7.

16 Sinha SK, Jha MJ, Mishra V, et al. Radial artery occlusion - incidence, predictors and long-term outcome after transradial catheterization: clinico-Doppler ultrasound-based study (RAIL-TRAC study). Acta Cardiol 2017;72:318-27.

17 Valsecchi 0, Vassileva A, Cereda AF, et al. Early clinical experience with right and left distal transradial access in the anatomical snuffbox in 52 consecutive patients. $J$ Invasive Cardiol 2018:30:218-23.

18 Babunashvili A. TCT-810 Novel distal transradial approach for coronary and peripheral interventions. J Am Coll Cardiol 2018;72:B323.

19 Al-Azizi KM, Lotfi AS. The distal left radial artery access for coronary angiography and intervention: A new era. Cardiovasc Revasc Med 2018;19:35-40.

20 Soydan E, Akın M. Coronary angiography using the left distal radial approach - an alternative site to conventional radial coronary angiography. Anatol J Cardiol 2018;19:243-8.

21 McCarthy DJ, Chen SH, Brunet MC, et al. Distal radial artery access in the anatomical snuffbox for neurointerventions: case report. World Neurosurg 2019;122:355-9.

22 Snelling BM, Sur S, Shah SS, et al. Transradial cerebral angiography: techniques and outcomes. J Neurointerv Surg 2018;10:874 881.

23 Snelling BM, Sur S, Shah SS, et al. Transradial access: lessons learned from cardiology. J Neurointerv Surg 2018;10:487-92.

24 Sur S, Snelling B, Khandelwal P, et al. Transradial approach for mechanical thrombectomy in anterior circulation large-vessel occlusion. Neurosurg Focus 2017;42:E13.

25 Chen SH, Snelling BM, Sur S, et al. Transradial versus transfemoral access for anterior circulation mechanical thrombectomy: comparison of technical and clinical outcomes. J Neurointerv Surg 2019:doi: neurintsurg-2018-014485.

26 Haussen DC, Nogueira RG, DeSousa KG, et al. Transradial access in acute ischemic stroke intervention. J Neurointerv Surg 2016:8:247-50. 
27 Barbeau GR, Arsenault F, Dugas L, et al. Evaluation of the ulnopalmar arterial arches with pulse oximetry and plethysmography: comparison with the Allen's test in 1010 patients. Am Heart J 2004;147:489-93.

28 Doscher W, Viswanathan B, Stein T, et al. Hemodynamic assessment of the circulation in 200 normal hands. Ann Surg 1983;198:776-9.

29 Seto AH, Roberts JS, Abu-Fadel MS, et al. Real-time ultrasound guidance facilitates transradial access: RAUST (Radial Artery access with Ultrasound Trial). JACC Cardiovasc Interv 2015:8:283-91.
30 Abualayem M, Hawatmeh A, Shittu M, et al. Aberrant right subclavian artery (arteria lusoria) diagnosed during transradial coronary catheterization. Quant Imaging Med Surg 2016;6:328-30.

31 Kaledin AL, Kochanov IN, Seletskiı̌ SS, et al. [Peculiarities of arterial access in endovascular surgery in elderly patients]. Adv Gerontol 2014;27:115-9.

32 Koutouzis M, Kontopodis E, Tassopoulos A, et al. Distal versus traditional radial approach for coronary angiography. Cardiovasc Revasc Med 2018. 